\title{
Optimización en el Manejo de Reproductores para una Mayor Producción de Huevos y Larvas de Psetta maxima (Linneaus, 1758)
}

\author{
Optimization of Broodstock Management to Increase the Production \\ of Eggs and Larvae of Psetta maxima (Linneaus, 1758) (Turbot)
}

\author{
Renzo Pepe-Victoriano ${ }^{*, * *}$; Miguel Araya ${ }^{* * *, * * * *}$; Víctor Faúndez ${ }^{* * * * * *}$ \& Manuel Rodríguez ${ }^{* * * * * * *}$
}

PEPE-VICTORIANO, R.; ARAYA, M.; FAÚNDEZ, V. \& RODRÍGUEZ, M. Optimización en el manejo de reproductores para una mayor producción de huevos y larvas de Psetta maxima (Linneaus, 1758). Int. J. Morphol., 31(3):873-878, 2013.

RESUMEN: Las óptimas condiciones de cultivo en el manejo de reproductores de Psetta maxima permiten obtener una mejor cantidad y calidad de huevos así como larvas viables con una mayor producción. Para evaluar las óptimas condiciones se utilizaron ejemplares mantenidos en condiciones de cultivo acondicionados desde los 3 hasta los 24 meses de vida, para así llegar a conformar un stock de reproductores. Al final de la experiencia, los huevos en los estanques experimentales se presentaron circulares y transparentes, al igual que las larvas recién eclosionadas que se hallaban rectas y muy bien pigmentadas, signos de buena calidad de huevos y larvas. Los cambios en la relación macho-hembra y densidad, fueron las variables a evaluar, llegando a obtener resultados satisfactorios por periodo productivo de las hembras y una mejor calidad y cantidad de huevos y larvas viables, en un periodo de cuatro años. Las mortalidades de reproductores en el periodo desove y post-desove bajaron significativamente. Estadísticamente no existe una diferencia significativa entre los estanques experimentales y controles. Desde el tercer año, la producción de huevos y larvas en los estanques experimentales mostró un incremento cercano al $90 \%$ en huevos y a un $60 \%$ en larvas. En los dos últimos años el porcentaje de larvas cosechadas en estos estanques, mostró un incremento del $7 \%$ al $12 \%$ en larvas sembradas y una disminución del $9 \%$ al $13 \%$ en larvas eliminadas, principalmente en el estanque 4.

PALABRAS CLAVE: Turbot; Huevos de peces; Larvas de peces

\section{INTRODUCCIÓN}

Para optimizar la calidad y cantidad de ovas en reproductores de turbot en los centros de cultivos productivos, es importante determinar las mejores condiciones en la mantención de estos, y obtener así un mejor desove. Actualmente hay diversidad de información dispersa que indican aplicaciones para el acondicionamiento de los reproductores como lo indican Iglesias et al. (1995), Peleteiro et al. (1995) y Dhert et al. (1999).

El correcto manejo de los reproductores y estrategias de desove son los factores que influyen en la calidad de huevos y larvas (Minkoff \& Broadhurst, 1994; Estévez et al., 1999), los cuales llevan a obtener buenas condiciones para el cultivo de estos (Kuhlman \& Quantz, 1980; Jones, 1989).
Peleteiro (1999) postula que las condiciones extremas en densidad y volúmenes de estanque dificulta la obtención de puestas naturales a pesar que en estas condiciones tanto las hembras como los machos realizan su ciclo de maduración naturalmente.

Un fotoperíodo de 16 horas de luz, produce maduración y desoves por un período de 2 a 3 meses, el cual radica en una producción de huevos de buena calidad con un similar porcentaje de fertilización y eclosión, comparado con otros desoves con fotoperíodo natural (Forés et al., 1990).

Se ha demostrado que el estado de nutrición de los reproductores puede afectar la calidad de la descendencia,

\footnotetext{
Centro de Recursos Naturales y Medio Ambiente (CERENAYMA), Universidad de Tarapacá, Arica, Chile.

** Programa de Doctorado en Ciencias, Mención Biología. Universidad de Tarapacá, Arica, Chile.

*** Facultad de Recursos Naturales Renovables, Universidad Arturo Prat, Iquique, Chile.

**** Programa de Doctorado en Ciencias Aplicadas Mención Sistemas Marinos Costeros, Universidad de Antofagasta, Antofagasta, Chile.

******* Departamento de Ingeniería Ambiental y Recursos Naturales, Universidad Católica de la Santísima Concepción, Concepción, Chile.

******* Departamento de Agronomía, Universidad de Tarapacá, Arica, Chile.
} 
en especial atención a la garantía óptima de sobrevivencia y desarrollo de la larva durante el periodo de alimentación endógena (Lavens et al., 1999).

La fecundidad ha sido usada para determinar la calidad de huevos, quien es también afectada por la deficiencia nutricional en la dieta de los reproductores (Izquierdo et al., 2001). La mayor parte de las especies, cuando se mantienen en cautiverio y en buenas condiciones, producen huevos de mejor calidad que los reproductores salvajes, esto es producto de las condiciones ambientales y alimentación a que están sometidos en cautiverio.

En el presente trabajo se evaluó el desarrollo de las condiciones óptimas de cultivo y manejo en reproductores de Psetta maxima, bajo los parámetros de relación machohembra y densidad, con el objetivo de una mejor producción de huevos y larvas.

\section{MATERIAL Y MÉTODO}

Se utilizaron ejemplares Psetta maxima de $20 \mathrm{~g}$, mantenidos en cautividad en las dependencias de una empresa comercial de turbot, los que fueron mantenidos por un período de dos años, en condiciones adecuadas de cultivo, según protocolo de producción de la empresa, para la generación de futuros reproductores, proporcionándoles un tratamiento distinto que los peces de engorda.

Para una mejor optimización del proceso reproductivo del cultivo de turbot, fue necesario realizar cambios al protocolo de origen, el cual consistió en modificar la relación macho-hembra y la densidad de cultivo.

Cuando se observaron sus primeras señales de maduración (tercer verano de vida), los peces seleccionados se distribuyeron en nueve estanques circulares (Tabla I), construidos en fibra de vidrio de 4 metros de diámetro y un metro de altura.
Efectuadas las distribuciones de los ejemplares reproductores, se mantuvieron en los estanques números 1,2 y 3 las mismas condiciones de los años anteriores, considerando estos estanques como grupo control, en los estanques 4, 5 y 6 , la diferencia con el grupo control fue la variable "relación macho-hembra" y en los estanques 7, 8 y 9 la variable diferente fue "la densidad de cultivo" (Tabla II).

El rango de peso de los peces en los estanques 1, 2 y 3 fue de 1750 y $2050 \mathrm{~g}$, mientras que en los estanques 4, 5, 6 y $7,8,9$, el rango fue de 1340 y $2890 \mathrm{~g}$.

El cambio de la relación macho-hembra fue alterado en los estanques experimentales. El manejo de esta medida implicó cambio de ejemplares, como aquellos que no cumplían con los requerimientos de peso (Tabla II), pigmentación, deformaciones y producción de gametos viables.

Tal como en el punto anterior, la densidad de cultivo de reproductores no se encontraba dentro de los parámetros descritos para esta especie $\left(12 \mathrm{a} 15 \mathrm{~kg} / \mathrm{m}^{2}\right)$, por lo que fue necesario incorporar nuevos especímenes de menor peso y extraer algunos de mayor peso, para así ajustar a la densidad óptima de esta especie (Tabla II).

Para el manejo óptimo de los reproductores fue necesario insistir en el cuidado extremo que se debe tener con estos peces, ya que un mal manejo podría causar daños físicos y estrés, que puede ocasionar la regresión rápida de las gónadas con lo que se pierde el desove.

\section{RESULTADOS}

La cantidad de huevos y larvas sembradas en los estanques experimentales va en aumento en los últimos dos años, para ambas variables en estudio, relación macho/hembra y densidad (Fig. 1).

Tabla I. Distribución de los peces en los estanques experimentales, anterior al inicio del experimento.

\begin{tabular}{lcccccc}
\hline & $\begin{array}{c}\text { Número } \\
\text { machos }\end{array}$ & Biomasa kg & $\begin{array}{c}\text { Número } \\
\text { hembras }\end{array}$ & Biomasa kg & $\begin{array}{c}\text { Relación } \\
\text { macho/hembra }\end{array}$ & $\begin{array}{c}\text { Densidad } \\
\mathrm{kg} / \mathrm{mt} 2\end{array}$ \\
\hline Estan. 1 & 21 & 41 & 22 & 43 & $1,0 / 0,96$ & 6,68 \\
Estan. 2 & 23 & 46 & 23 & 47 & $1,0 / 1,0$ & 7,40 \\
Estan. 3 & 23 & 47 & 24 & 49 & $1,0 / 0,96$ & 7,59 \\
Estan. 4 & 22 & 41 & 22 & 44 & $1,0 / 1,0$ & 6,76 \\
Estan. 5 & 24 & 47 & 23 & 50 & $1,0 / 1,04$ & 7,72 \\
Estan. 6 & 24 & 46 & 24 & 51 & $1,0 / 1,0$ & 7,91 \\
Estan. 7 & 25 & 42 & 23 & 49 & $1,0 / 1,09$ & 7,24 \\
Estan. 8 & 24 & 44 & 23 & 51 & $1,0 / 1,04$ & 7,56 \\
Estan. 9 & 23 & 45 & 24 & 50 & $1,0 / 0,96$ & 7,56 \\
\hline
\end{tabular}


Tabla II. Distribución de los peces en los estanques experimentales (4, 5 y 6 relación macho/hembra; 7, 8 y 9 densidad de cultivo) posterior a las modificaciones.

\begin{tabular}{|c|c|c|c|c|c|c|}
\hline $\begin{array}{l}\text { Estanque } \\
\text { Número } 4\end{array}$ & $\begin{array}{l}\text { Número de } \\
\text { machos }\end{array}$ & $\begin{array}{l}\text { Biomasa } \\
(\mathrm{kg})\end{array}$ & $\begin{array}{l}\text { Número de } \\
\text { Hembras }\end{array}$ & $\begin{array}{c}\text { Biomasa } \\
(\mathrm{kg})\end{array}$ & $\begin{array}{c}\text { Relación } \\
\text { macho/hembra }\end{array}$ & $\begin{array}{c}\text { Densidad } \\
\left(\mathrm{kg} / \mathrm{m}^{2}\right)\end{array}$ \\
\hline Ago. 2002 & 42 & 90,3 & 31 & 70,9 & $1 / 1.35$ & 12,83 \\
\hline Ago. 2003 & 42 & 70,3 & 31 & 56,3 & $1 / 1.35$ & 10,08 \\
\hline Ago. 2004 & 42 & 78,3 & 31 & 89,5 & $1 / 1.35$ & 13,36 \\
\hline Ago. 2005 & 42 & 86,5 & 31 & 90,2 & $1 / 1.35$ & 14,07 \\
\hline $\begin{array}{l}\text { Estanque } \\
\text { Número } 5\end{array}$ & $\begin{array}{l}\text { Número de } \\
\text { machos }\end{array}$ & $\begin{array}{l}\text { Biomasa } \\
(\mathrm{kg})\end{array}$ & $\begin{array}{l}\text { Número de } \\
\text { Hembras }\end{array}$ & $\begin{array}{l}\text { Biomasa } \\
(\mathrm{kg})\end{array}$ & $\begin{array}{c}\text { Relación } \\
\text { macho/hembra }\end{array}$ & $\begin{array}{c}\text { Densidad } \\
\left(\mathrm{kg} / \mathrm{m}^{2}\right)\end{array}$ \\
\hline Ago. 2002 & 42 & 89,9 & 31 & 56,8 & $1 / 1.35$ & 11,68 \\
\hline Ago. 2003 & 42 & 69,5 & 31 & 49,3 & $1 / 1.35$ & 9,46 \\
\hline Ago. 2004 & 42 & 98,2 & 31 & 49,2 & $1 / 1.35$ & 11,74 \\
\hline Ago. 2005 & 42 & 89,2 & 31 & 89,2 & $1 / 1.35$ & 14,20 \\
\hline $\begin{array}{l}\text { Estanque } \\
\text { Número } 6\end{array}$ & $\begin{array}{l}\text { Número de } \\
\text { machos }\end{array}$ & $\begin{array}{c}\text { Biomasa } \\
(\mathrm{kg})\end{array}$ & $\begin{array}{l}\text { Número de } \\
\text { Hembras }\end{array}$ & $\begin{array}{c}\text { Biomasa } \\
(\mathrm{kg})\end{array}$ & $\begin{array}{c}\text { Relación } \\
\text { macho/hembra }\end{array}$ & $\begin{array}{c}\text { Densidad } \\
\left(\mathrm{kg} / \mathrm{m}^{2}\right)\end{array}$ \\
\hline Ago. 2002 & 42 & 95,5 & 31 & 65,2 & $1 / 1.35$ & 12,79 \\
\hline Ago. 2003 & 42 & 82,2 & 31 & 53,1 & $1 / 1.35$ & 10,77 \\
\hline Ago. 2004 & 42 & 56,1 & 31 & 59,9 & $1 / 1.35$ & 9,24 \\
\hline Ago. 2005 & 42 & 59,8 & 31 & 60,1 & $1 / 1.35$ & 9,55 \\
\hline $\begin{array}{l}\text { Estanque } \\
\text { Número } 7\end{array}$ & $\begin{array}{l}\text { Número de } \\
\text { machos }\end{array}$ & $\begin{array}{c}\text { Biomasa } \\
(\mathrm{kg})\end{array}$ & $\begin{array}{l}\text { Número de } \\
\text { Hembras }\end{array}$ & $\begin{array}{l}\text { Biomasa } \\
(\mathrm{kg})\end{array}$ & $\begin{array}{c}\text { Relación } \\
\text { macho/hembra }\end{array}$ & $\begin{array}{c}\text { Densidad } \\
\left(\mathrm{kg} / \mathrm{m}^{2}\right)\end{array}$ \\
\hline Ago. 2002 & 39 & 87,2 & 33 & 65,3 & $1 / 1.18$ & 12,14 \\
\hline Ago. 2003 & 35 & 83,2 & 36 & 64,2 & $1 / 0,97$ & 11,74 \\
\hline Ago. 2004 & 38 & 84,6 & 28 & 61,9 & $1 / 1,36$ & 11,66 \\
\hline Ago. 2005 & 40 & 86,9 & 29 & 62,3 & $1 / 1,8$ & 11,88 \\
\hline $\begin{array}{l}\text { Estanque } \\
\text { Número } 8\end{array}$ & $\begin{array}{l}\text { Número de } \\
\text { machos }\end{array}$ & $\begin{array}{c}\text { Biomasa } \\
(\mathrm{kg})\end{array}$ & $\begin{array}{l}\text { Número de } \\
\text { Hembras }\end{array}$ & $\begin{array}{c}\text { Biomasa } \\
(\mathrm{kg})\end{array}$ & $\begin{array}{c}\text { Relación } \\
\text { macho/hembra }\end{array}$ & $\begin{array}{c}\text { Densidad } \\
\left(\mathrm{kg} / \mathrm{m}^{2}\right)\end{array}$ \\
\hline Ago. 2002 & 48 & 85,3 & 25 & 63,2 & $1 / 1,92$ & 11,82 \\
\hline Ago. 2003 & 45 & 84,2 & 29 & 65,5 & $1 / 1.55$ & 11,92 \\
\hline Ago. 2004 & 39 & 83,6 & 38 & 61,6 & $1 / 1.03$ & 11,56 \\
\hline Ago. 2005 & 50 & 89,2 & 31 & 63,9 & $1 / 1 ., 61$ & 12,19 \\
\hline $\begin{array}{c}\text { Estanque } \\
\text { Número } 9\end{array}$ & $\begin{array}{l}\text { Número de } \\
\text { machos }\end{array}$ & $\begin{array}{c}\text { Biomasa } \\
(\mathrm{kg})\end{array}$ & $\begin{array}{l}\text { Número de } \\
\text { Hembras }\end{array}$ & $\begin{array}{c}\text { Biomasa } \\
(\mathrm{kg})\end{array}$ & $\begin{array}{c}\text { Relación } \\
\text { macho/hembra }\end{array}$ & $\begin{array}{c}\text { Den sidad } \\
\left(\mathrm{kg} / \mathrm{m}^{2}\right)\end{array}$ \\
\hline Ago. 2002 & 40 & 84,2 & 30 & 62,3 & $1 / 1.33$ & 11,66 \\
\hline Ago. 2003 & 38 & 86,2 & 29 & 59,9 & $1 / 1.31$ & 11,63 \\
\hline Ago. 2004 & 49 & 88,9 & 28 & 64,0 & $1 / 1.75$ & 12,17 \\
\hline Ago. 2005 & 42 & 90,1 & 38 & 60,2 & $1 / 1.11$ & 11,97 \\
\hline
\end{tabular}

Los huevos en los estanques experimentales de los años 3 y 4 son circulares y transparentes, con un único glóbulo de aceite, de mayor tamaño a los obtenidos en los primeros años de la investigación $(1,2-1,5 \mathrm{~mm})$, el cual implica una mejor calidad de huevo, con una mayor flotabilidad. Estos se presentaron con un desarrollo regular en un porcentaje mayor al 90\%, comenzando con la expulsión del primer cuerpo polar, seguido de la segmentación hasta alcanzar el estado de mórula. Transcurridas 42 horas de la fecundación, el embrión ocupa la semicircunferencia del huevo, a las 90 horas el embrión presenta una leve pigmentación, a las 102 horas el embrión está prácticamente formado, presenta movimientos esporádicos y se puede observar los latidos cardiacos. A las 115 horas aproximadamente se rompe el corion, liberándose una larva de 2,7 a 3,1 mm de longitud.

La relación macho/hembra fue constante, la biomasa de cada uno de ellos tuvo una variación producto de las mortalidades producidas en el periodo post desove, que conlleva al reemplazo de estos peces en el próximo periodo de invierno.

Las densidades de cultivo se mantuvieron entre el rango establecido para reproductores de esta especie, cercanas al rango inferior de $12 \mathrm{~kg} / \mathrm{m}^{2}$ (Tabla II), lo que permite el crecimiento de estos peces en el transcurso del año sin sobrepasar el rango máximo de $15 \mathrm{~kg} / \mathrm{m}^{2}$. 

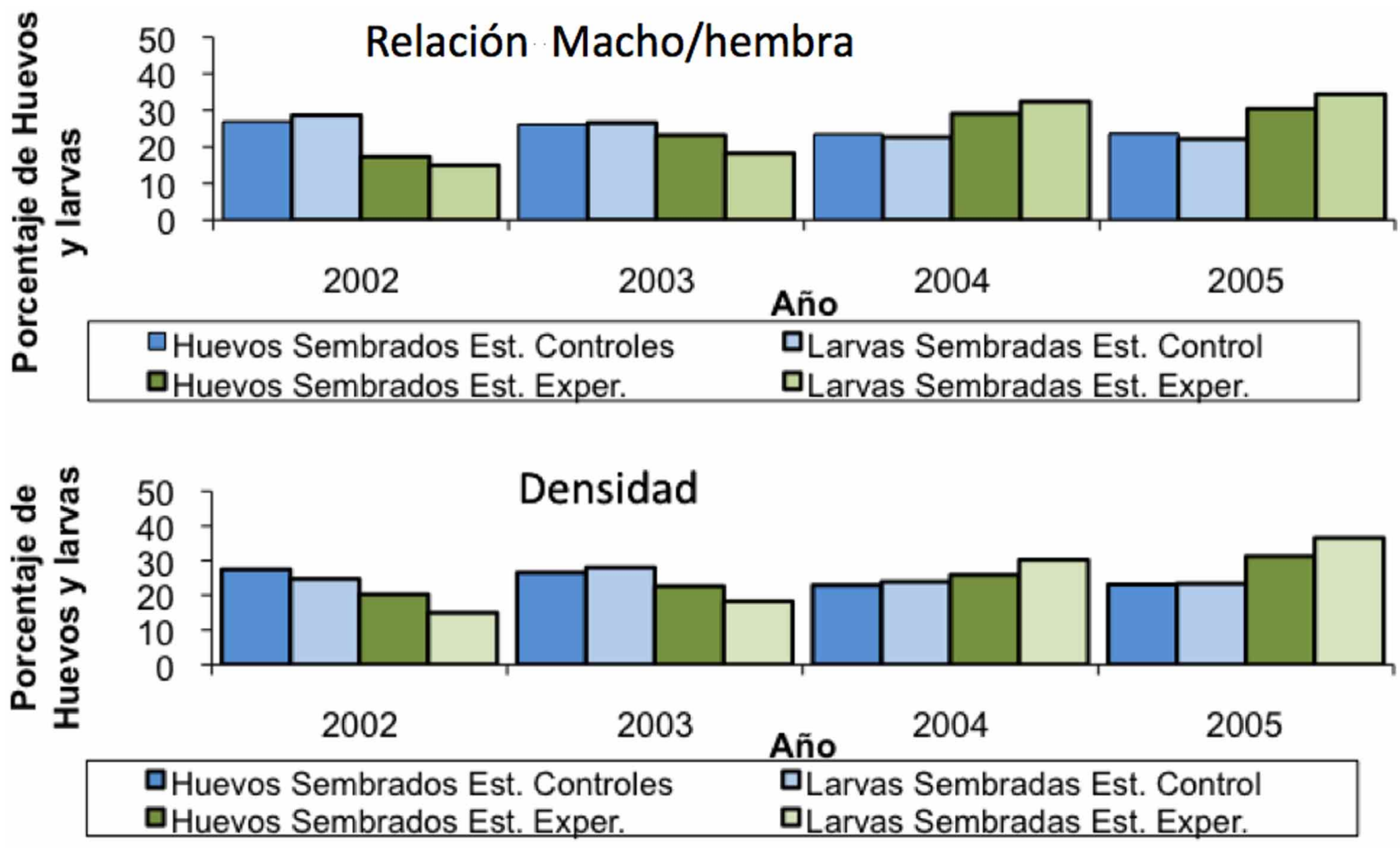

Fig. 1. Producción porcentual de huevos sembrados y larvas sembradas en los estanques controles y experimentales.

\section{$\%$ de mortalidad en el tiempo}

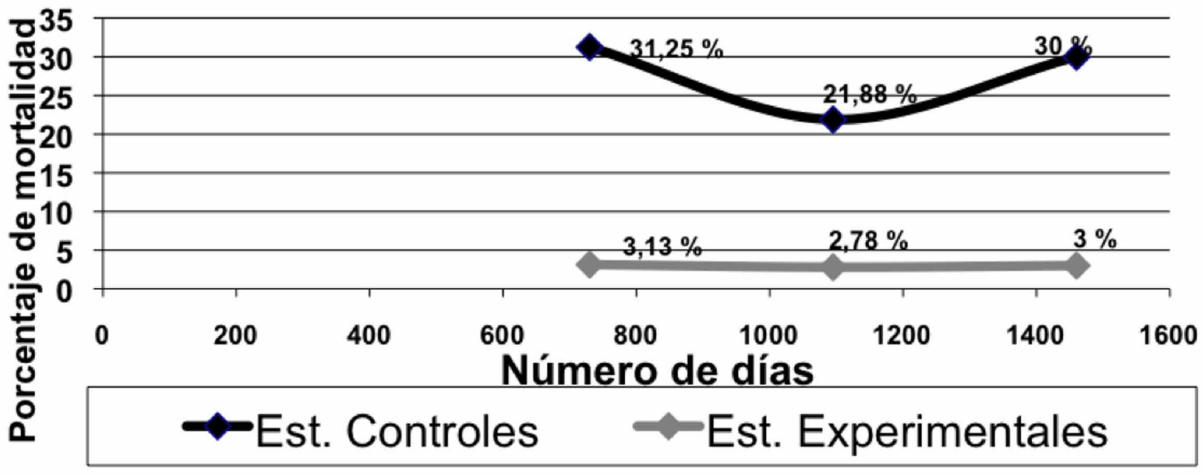

Fig. 2. Mortalidad en los estanques reproductores, controles y experimentales.

No se encontraron diferencias significativas $(\mathrm{p}<$ $0,05)$ para la variable larvas entre los estanques controles y entre los experimentales, a pesar de que en términos absolutos, descriptivamente, se observa un aumento del número de larvas viables a contar del tercer año de manejo.

Para la variable reproductores muertos, si se encon- traron diferencias entre las proporciones estimadas de estanques controles y experimentales, es decir la tasa de mortalidad fue significativamente menor $(\mathrm{p}<0,05)$, cuando se aplica el manejo experimental propuesto. Esto es palpable a partir del tercer año de trabajo y con un porcentaje promedio de mortalidad del $\quad 27,71 \%$ en el caso de los estanques controles y baja ostensiblemente, 10 veces menos $(2,97 \%)$ en el caso de los estanques experimentales (Fig. 2). 


\section{DISCUSIÓN}

La gran demanda de la producción de peces marinos y las diversas variedades de especies que están constantemente incrementando la productividad acuícola, debido a las nuevas especies de alto valor comercial que se están introduciendo en este rubro, ha generado en los últimos años un interés masivo en la obtención de cantidad y calidad de huevos, en los hatcherys comerciales (Alvarez-Lajonchère et al., 2007) e instituciones particulares y universitarias, que han originado variedades de líneas de investigación. Tal conocimiento, ha generado el estudio de los reproductores de especies con alto valor comercial, principalmente en el área de la nutrición, lo cual conlleva a practicar otros vectores de trabajo, como la relación entre machos y hembras y la densidad como se ha analizado en el presente trabajo.

Los desoves mejoraron notablemente al final de la experiencia, esto radica en una puesta fácil y rápida en la expulsión de las ovas y líquido espermático, lo cual lleva además a una pronta recuperación de los reproductores post desove. Esto es atribuido a la sincronización del termoperiodo con el ciclo ovulatorio de la especie, que permite realizarlo sin dificultad.

La relación macho-hembra constituye un elemento fundamental en la dinámica poblacional y reproductiva de las especies (Avilés, 2005). La proporción más adecuada y con resultados positivos arrojados en este trabajo fue de 1,35:1 (macho-hembra) lo cual no fue concordante con resultados entregados por otros autores. En cambio Fauvei et al. (1993), reporta una proporción sexual de 0,8:1 (macho-hembra) para reproductores de turbot en Francia, el cual aún está lejos de nuestros resultados. En otras especies, estos fueron de 1,5:1 (macho-hembra) en la época de reproducción máxima y de 1:1 en el periodo de reposo o menor actividad reproductiva, lo cual se asemeja con los resultados nuestros y los de Sergio-Ferreira (1997) quien encontró una relación de 1,2:1 macho-hembra para la misma población.

La densidad de cultivo, escogida para los reproductores de turbot fue de 12 a $15 \mathrm{~kg} / \mathrm{m}^{2}$, lo cual se aleja bastante a lo reportado por Fauvei et al., quien señala una densidad de 5 a $6 \mathrm{~kg} / \mathrm{m}^{2}$.

Reconociendo que existe un control endógeno (luz y temperatura principalmente) del ciclo reproductivo estacional en turbot, los factores ambientales ejercen su influencia en cómo se desarrollara este ciclo en el tiempo. El ciclo reproductivo gonadal y la época de desove natural están modulados fundamentalmente por las variaciones del ciclo fotoperiodo anual; el patrón general de esta modulación implica que la recrudencia y el crecimiento gonadal comienzan al final del invierno y principios de primavera, estimulados por el fotoperiodo creciente de esa época del año (Díaz, 2006).

La temperatura juega un rol significativo en los estados finales de la maduración y desove (Pepe-Victoriano et al., 2012a), temperaturas bajas pueden inhibir la ovulación y temperaturas altas y constantes producen trastornos metabólicos en los ovocitos (Díaz).

Para la incubación artificial de huevos, se requiere de sistemas altamente puros y estables, una buena calidad de agua con alto contenido de oxigeno y baja en metabolitos; un flujo de agua constante con aireación permanente, que mantenga en movimiento los huevos sin dañarlos mecánicamente, son métodos a considerar para un buen desarrollo embriológico.

Las bajas tasas de eclosión, están asociadas a inmadurez espermática o de óvulos o bien a la asincronía en la maduración de ambos gametos (Pepe-Victoriano et al., 2012b), reflejando problemas en el manejo de foto o termoperíodo. Normalmente es el fotoperíodo el que gatilla el proceso de madurez gonadal pero la calidad depende del termoperíodo asociado. Además se debe tener la precaución de ajustar el nivel de aire tanto en cantidad como en dirección para evitar pérdida de larvas por aglomeración.

En plantas de producción la baja mortalidad es un requisito necesario, más no suficiente para una mayor puesta de huevos, ya que estos animales se reponen constantemente en los periodos de otoño-invierno de los estanques, por lo que siempre se asegura una cantidad de animales adecuadas para brindar el número de huevos y larvas suficientes, para así asegurar la producción anual comprometida.

Para la variable larvas entre los estanques controles y entre los experimentales, se puede interpretar entendiendo que a pesar de observarse un aumento del número de larvas viables a contar del tercer año de manejo, la variabilidad relativa es compartida, es decir existe traslape de desviaciones, luego es importante precisar los parámetros que permitan controlar esta variabilidad: densidad y relación macho/hembra.

Para la variable reproductores, entre los estanques controles y entre los experimentales la sobrevivencia de animales en el esquema propuesto en esta investigación fue mucho más alta que en el protocolo establecido, obteniendo menor mortalidad. Esto se debió principalmente a la densidad y relación macho/hembra. 
PEPE-VICTORIANO, R.; ARAYA, M.; FAÚNDEZ, V. \& RODRÍGUEZ, M. Optimization of broodstock management to increase the production of eggs and larvae of Psetta maxima (Linneaus, 1758) (Turbot). Int. J. Morphol., 31(3):873-878, 2013.

SUMMARY: The optimal culture conditions in broodstock management of Psetta maxima allow obtaining better quantity and quality of eggs and viable larvae, increasing the production. In order to evaluate the optimal conditions the specimens kept under culture conditions were used, in a period of 3-24 months of life, thus forming a broodstock stock. At the end of the experiment, the eggs in the experimental tanks resulted circular and transparent, as well as newly hatched larvae that were straight and well pigmented, signs of a good quality of eggs and larvae. Changes in the male-female and density ratio were the assessed variables, reaching satisfactory results per productive period of females and better quality and quantity of eggs and viable larvae during the period of four years. The mortality rate of broodstock in spawning and post-spawning period decreased significantly. Statistically there were no significant difference between experimental and control tanks. From the third year, egg and larvae production in experimental tanks showed an increase of about $90 \%$ for eggs, and $60 \%$ for larvae. In the last two years the percentage of harvested larvae in these tanks showed an increase of $7 \%$ to $12 \%$ for sown larvae and a decrease of $9 \%$ to $13 \%$ for eliminated larvae, mostly in tank 4 .

KEY WORDS: Turbot; Eggs of fish; Larvae of fish.

\section{REFERENCIAS BIBLIOGRÁFICAS}

Alvarez-Lanjonchère, L.; Reina Cañez, M. A.; Camacho-Hernández, M. A. \& Kraul, S. Design of a pilot-scale tropical marine finfish hatchery for a research center at Mazatlán, Mexico. Aquacult. Eng., 36(2):81-96. 2007.

Aviles, M. Calidad de huevos y larvas según el manejo de los reproductores de la cabrilla (Paralabrax maculatofasciatus), Pisces: Serranidae. Tesis para optar al grado de Doctor en Ciencias Biológicas, Universidad de Barcelona, Barcelona, 2005.

Dhert, P.; Gonzalez, F. M.; Van-Ryckeghem, K.; Geurden, I.; Thysen, F.; Lebegue, E.; Lavens, P. \& Sorgeloos, P. Cofeeding of phospholipids to turbot Scophthalmus maximus L. larvae as a tool to reduce live food consumption. Aquacult. Nut., 5(4):237-45, 1999.

Díaz, N. Manual de biotecnologías para el manejo reproductivo en peces. Facultad de Ciencias de la Universidad de Chile, Santiago, 2006.

Estévez, A.; McEvoy, L. A.; Bell, J. G. \& Sargent, J. R. Growth, survival, lipid composition and pigmentation of turbot (Scophthalmus maximus) larvae fed live-prey enriched in Arachidonic and Eicosapentaenoic acids. Aquaculture, 180(3-4):321-43, 1999.

Fauvei, C.; Omnes, M.; Mugnier, C.; Normant, Y.; Dorange, G. \& Suquet, M. La reproduction du turbot. Aspects biologiques et gestion dês reproducteurs. La Pisciculture Francaise, 112:23-40, 1993.

Forés, R.; Iglesias, J.; Olmedo, M.; Sánchez, F. \& Peleteiro, J. Induction of spawning in Turbot (Scophthalmus maximus L.) by a Sudden Change in the photoperiod. Aquacult. Eng., 9(5):357-66, 1990.

Iglesias, J.; Rodríguez, G. \& Peleteiro, J. Effect of light and temperature on the development of turbot eggs (Scophthalmus maximus L.). ICES Mar. Sci. Symp., 201:40-4, 1995.

Izquierdo, M. S.; Fernández-Palacios, H. \& Tacon, A. G. J. Effect of broodstock nutrition on reproductive performance of fish. Aquaculture, 197(1-4):25-42, 2001.

Jones, A. Incubation of turbot eggs Scophthalmus maximus. Publicacions do Seminario de Estudos Galegos. In: Iglesias, J. (Ed.). Cuadernos da Área de Ciências Marinas. Sada. A Coruña, Edicions do Castro, 1989. pp. 29-36.

Kuhlmann, D. \& Quantz, G. Some effects of temperature and salinity on the embryonic development and incubation time of the turbot Scophthalmus maximus L., from the Baltic Sea. Meeresforschung, 28(2):172-8, 1980.

Lavens, P.; Lebegue, E.; Jaunet, H.; Brunel, A.; Dhert, P. \& Sorgeloos, P. Effects of dietary essential fatty acids and vitamins on egg quality in turbot broodstocks. Aquacult. Int., 7:225-40, 1999.

Minkoff, G. \& Broadhurst, A. Intensive production of turbot, Scophthalmus maximus, fry. In: Lavens, P. \& Remmerswaal, R. A. M. (Eds.). Turbot culture: problems and prospects. Oostende, European Aquaculture Society, 1994. pp.14-31.

Peleteiro, J.; Lavens, P.; Rodriguez-Ojea, G. \& Iglesias, J. Relationship between egg quality and fatty acid content of various turbot broodstocks (Scophthalmus maximus L.). ICES Mar. Sci. Symp., 201:51-6, 1995.

Peleteiro, J. La reproducción del Rodaballo (Scophthalmus maximus L.) en cautividad. II Congreso Ibérico de Reproducción Anual. Libro de ponencias y Resúmenes. Lugo, Universidad Santiago de Compostela, 1999. pp.1-13.

Pepe-Victoriano, R.; Silva, A.; Vega, A.; Araya, M. \& Cornejo, L. Efecto del Aumento de la Temperatura, Frecuencia de Alimentación y Ración de Alimento en el Crecimiento de Juveniles de Turbot Psetta maxima. Int. J. Morphol., 30(3):902-7, 2012a.

Pepe-Victoriano, R.; Araya, M. \& Faúndez, V. Efecto de la Temperatura en la Supervivencia Embrionaria y Primeros Estadios Larvales de Psetta maxima. Int. J. Morphol., 30(4):1551-7, 2012 b.

Sergio-Ferreira, A. Ciclo reproductor de hembras de Paralabrax maculatofasciatus (Steindachner, 1868) y su importancia para la acuacultura. Tesis de Maestría, Universidad Autónoma de Baja California Sur, La Paz, Mexico, 1997.

\section{Dirección para Correspondencia: \\ Renzo Pepe-Victoriano \\ Centro de Recursos Naturales y Medio Ambiente (CERENAYMA) \\ Universidad de Tarapacá \\ Arica \\ CHILE}

Email: rpepe@uta.cl

Recibido : 12-02-2013

Aceptado: 19-05-2013 